\title{
Controlled Whole-body Vibration Training Reduces Risk of Falls in People with Multiple Sclerosis
}

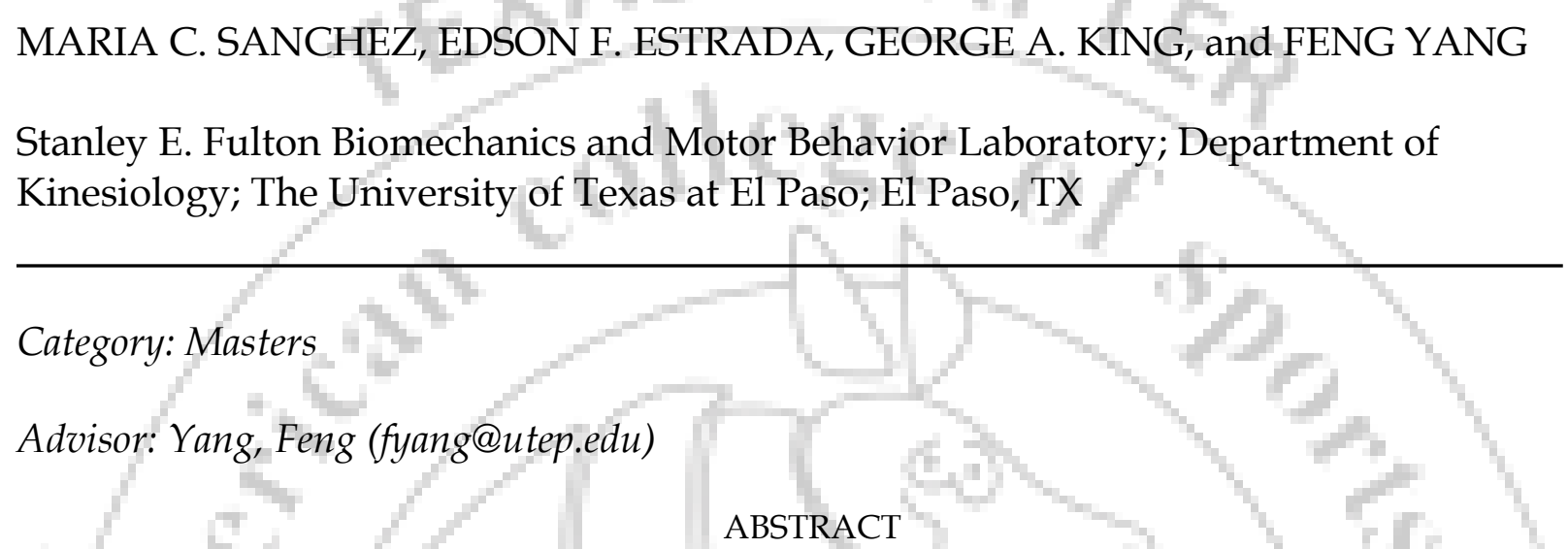

Despite the prevalence of falls among people with multiple sclerosis (MS), there are very limited evidencebased treatment approaches for fall prevention among this population. Controlled whole-body vibration (CWBV) training has recently emerged as a new modality to train older adults to reduce their risk of falls primarily because it is portable, safe, easy to use, and cost-effective; and requires less intensive physical activity than traditional exercise-based methods. Nevertheless, there is a lack of evidence about the impact of CWBV training on reducing falls in people with MS. The primary purpose of this study was to examine if an 8-week CWBV training protocol can reduce risk of falls in people with MS. Twenty-two individuals with MS (mean \pm standard deviation age: $52.0 \pm 13.7$ y/o; body height: $166.0 \pm 9.3 \mathrm{~cm}$; body mass: $79.5 \pm$ $24.4 \mathrm{~kg}$; disease duration: $15.5 \pm 11.1 \mathrm{y}$; Patient Determined Disability Step: $3.7 \pm 1.9$; 15 females) underwent the vibration training 3 times per week for 8 weeks while standing on a side alternating vibration platform with knees slightly bent. Each training session consisted of 5 repetitions of 1-min vibration exposure followed by a 1-min rest. The vibration frequency and amplitude were $20 \mathrm{~Hz}$ and 3.6 $\mathrm{mm}$, respectively. Their risk of falls was quantified by the isometric knee extensor muscle strength capacity (normalized to the body mass), the EquiScale body balance scale, and the functional mobility measured by the Timed-Up-and-Go (TUG) test. All risk factors were evaluated prior to (pre-training) and after (post-training) the 8 -week training. Paired $t$-tests were used to examine if the training improves these risk factors. Our results revealed that the knee extensor strength capacity on the weak side increased significantly form $0.88 \pm 0.45 \mathrm{Nm} / \mathrm{kg}$ for the pre-training test to $1.02 \pm 0.50 \mathrm{Nm} / \mathrm{kg}$ for the post-training test $(p<0.001)$. Compared to pre-training values, there was a significant improvement at post-training for both body balance $(11.1 \pm 4.0$ vs. $12.7 \pm 3.2, p<0.001)$ and TUG $(14.8 \pm 6.6$ vs. $13.0 \pm 5.5 \mathrm{sec}, p<0.01)$. The finding of this study suggested that an 8-week CWBV training program can be well accepted by people with MS. Our results supported that CWBV intervention could reduce the risk of falls among individuals with MS by strengthening their lower limb muscles, improving the body balance, and enhancing the functional mobility. The clinical application of CWBV may reduce falls for individuals with MS; however, more systematic studies based on large sample sizes are needed to investigate the longitudinal effects of vibration training to prevent real-life falls among persons living with MS. Supported by NMSS Grant PP-3385 (FY) 